\title{
Two decades of Antarctic coastal-change revealed by satellite imagery and deep learning
}

Celia Baumhoer ${ }^{1}$, Andreas Dietz ${ }^{1}$, Mariel Dirscherl ${ }^{1}$ and Claudia Kuenzer ${ }^{1,2}$ ${ }^{1}$ German Aerospace Center (DLR), Earth Observation Center (EOC), Oberpfaffenhofen, Germany

¿University Wuerzburg, Institute of Geography and Geology, Wuerzburg, Germany

Link to publication

$\underset{2021}{\text { EGUU General }}$

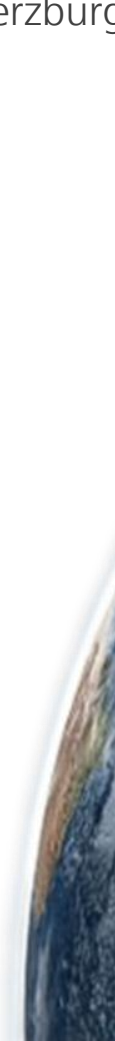




\section{Antarctic Coastline Extraction}

Implemented Processing Chain

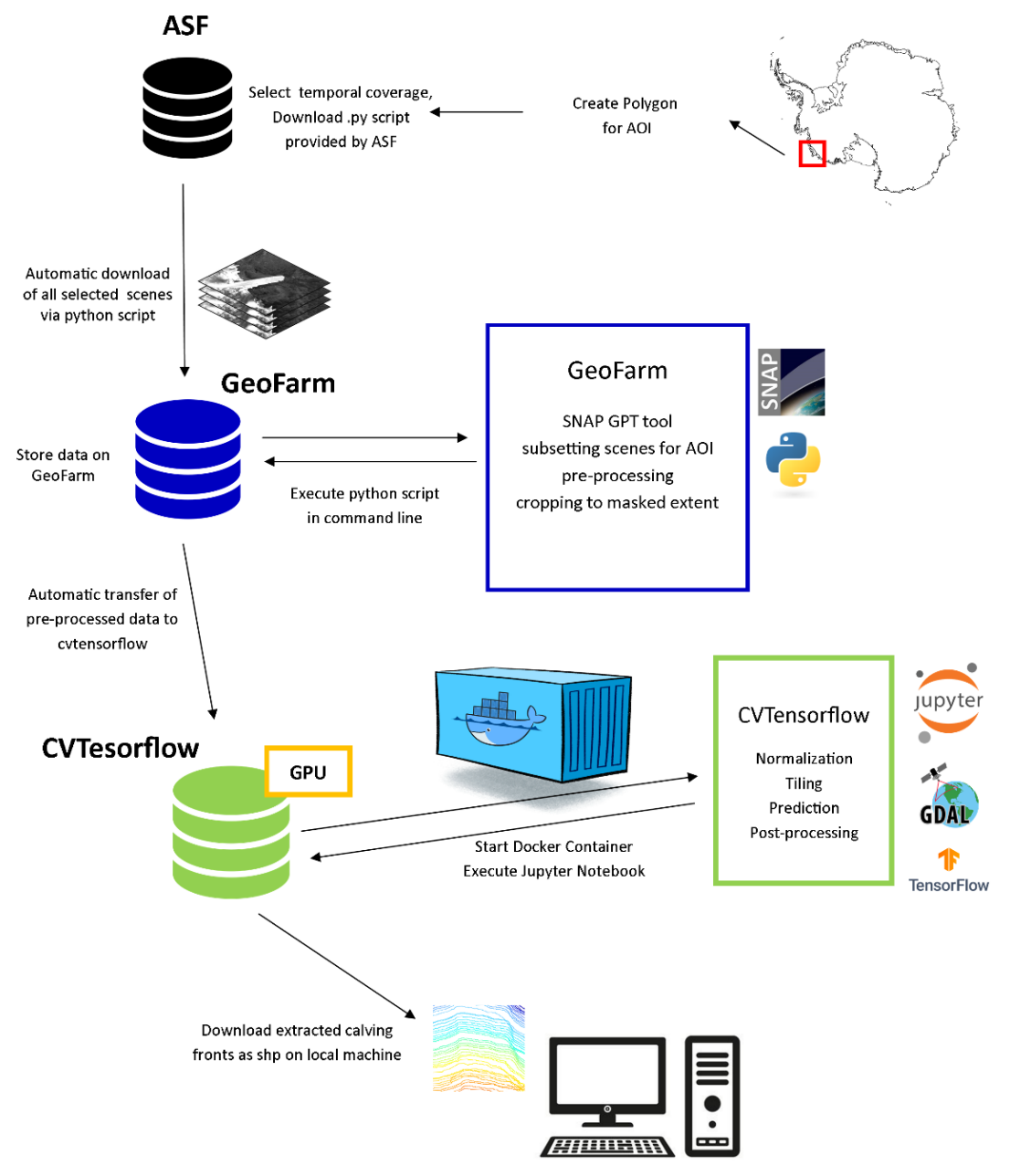

Sentinel-1 Data Coverage in total 175 Scenes

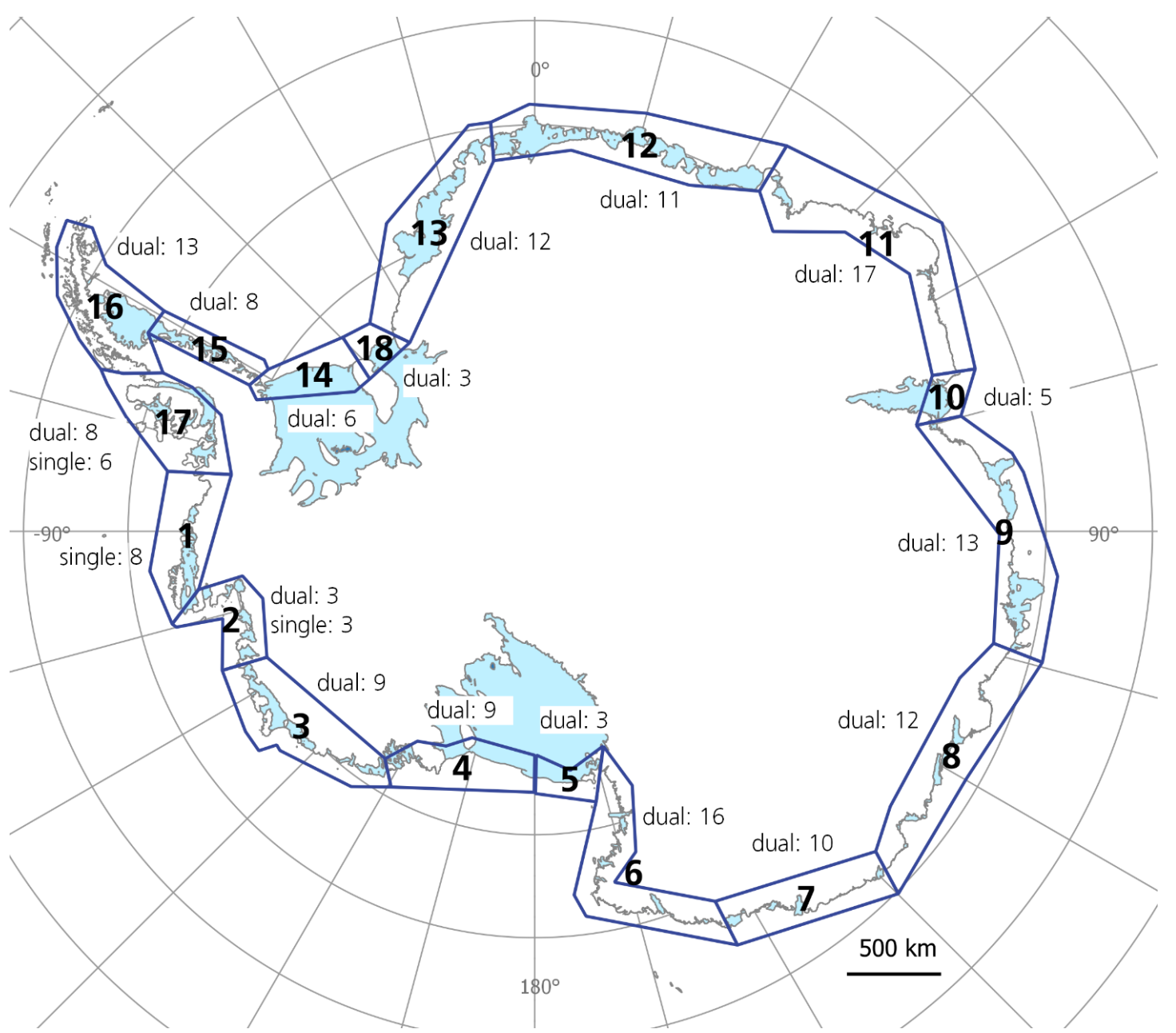




\section{Antarctic Coastline 2018}

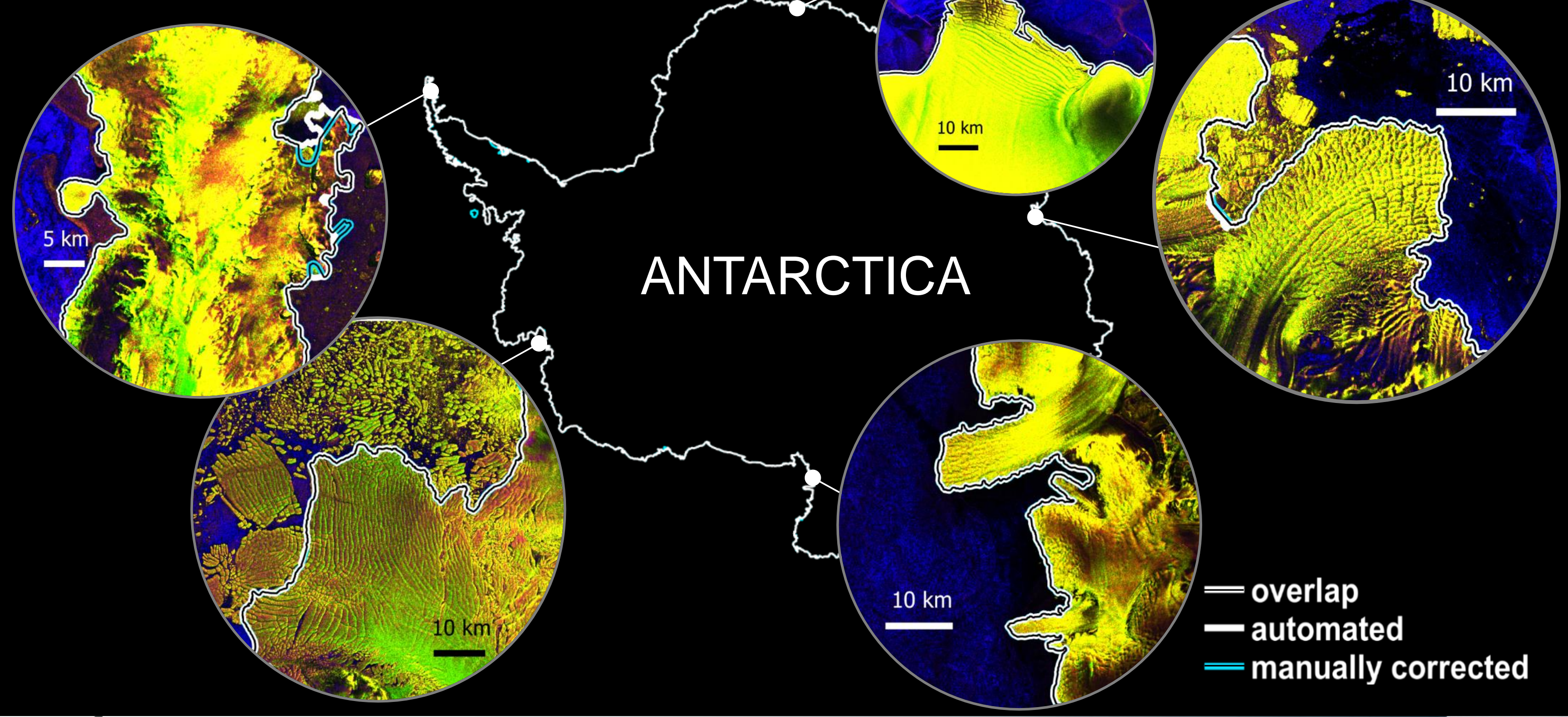




\section{Calculating Coastal-Change}

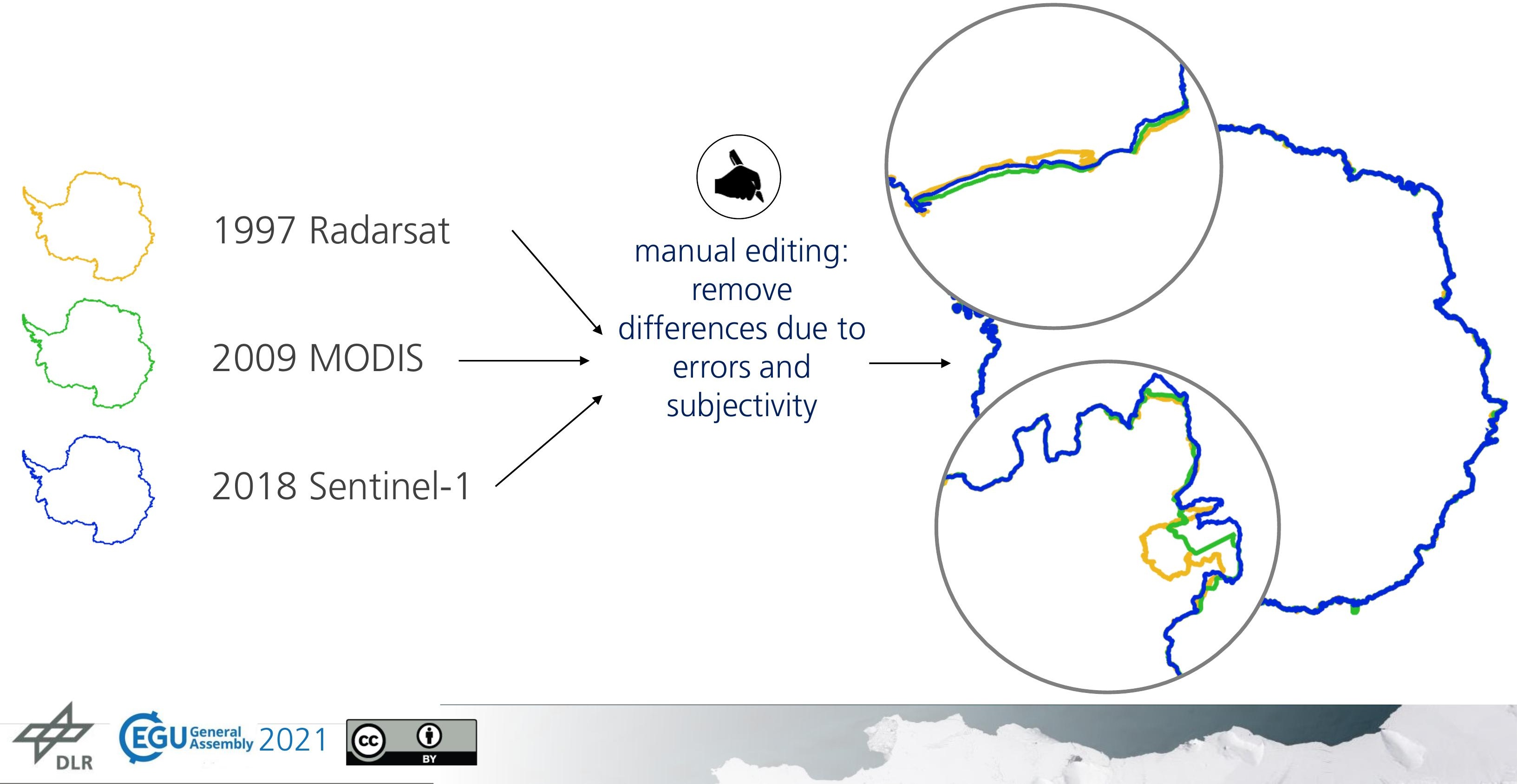




\section{Two Decades of Coastal-Change}

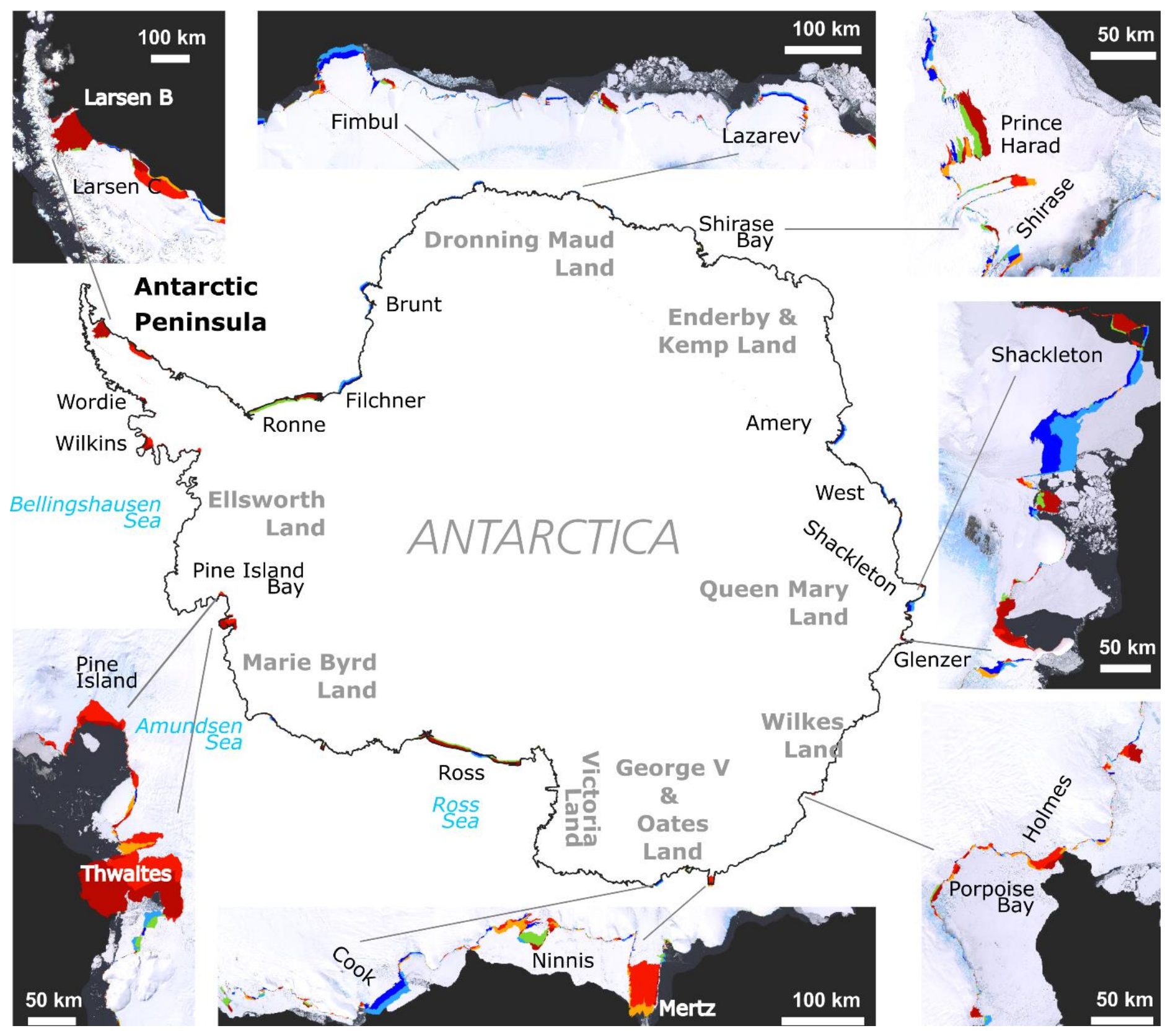

50 km 


\section{Glacier and Ice Shelf Front Change}

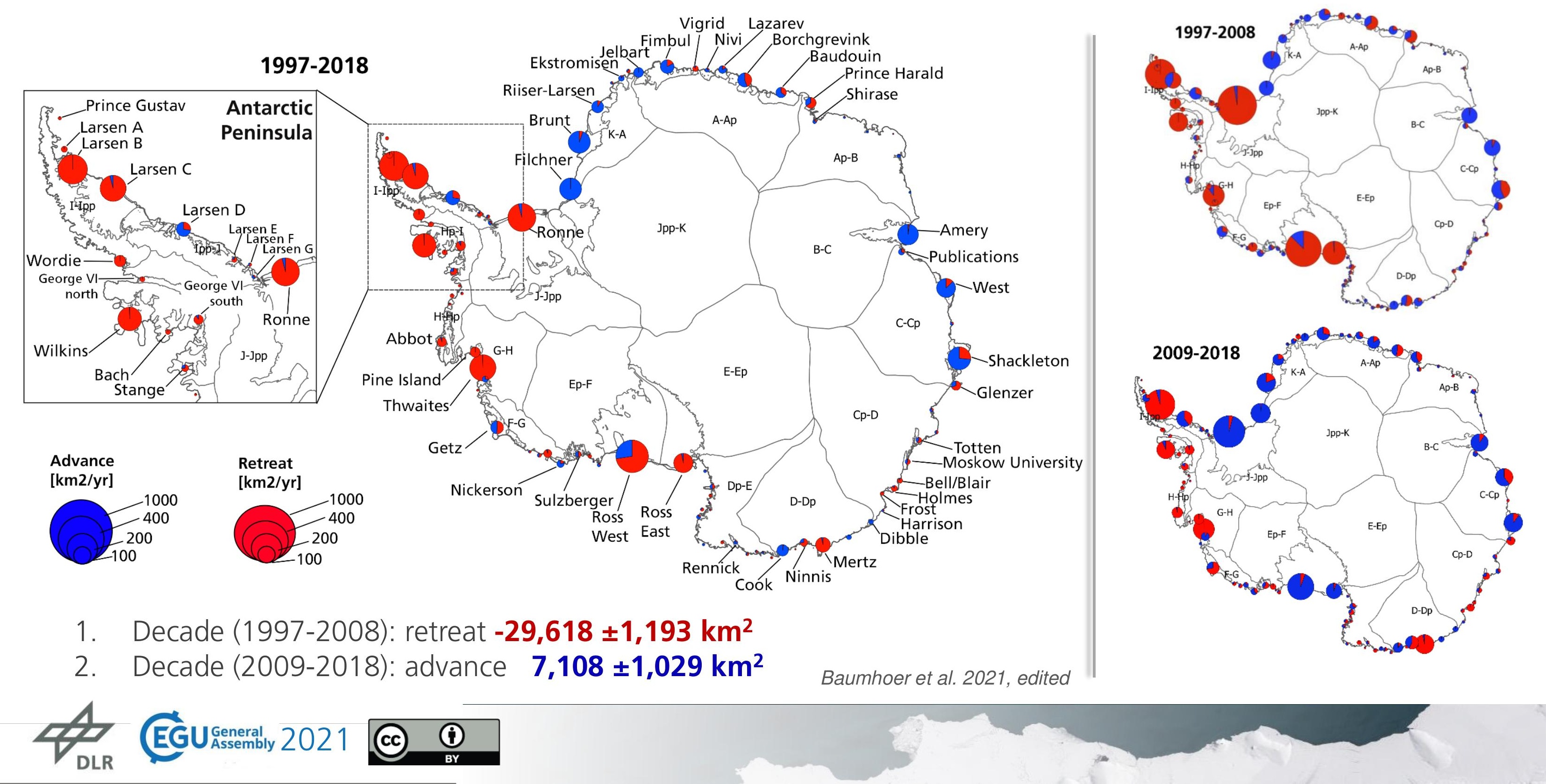




\section{Thank you for your interest!}

Calving at Brunt /ce Shelf (iceberg A-74) extracted coastline in white 26. Februar 2021

(C) Copernicus Sentinel-1 Data
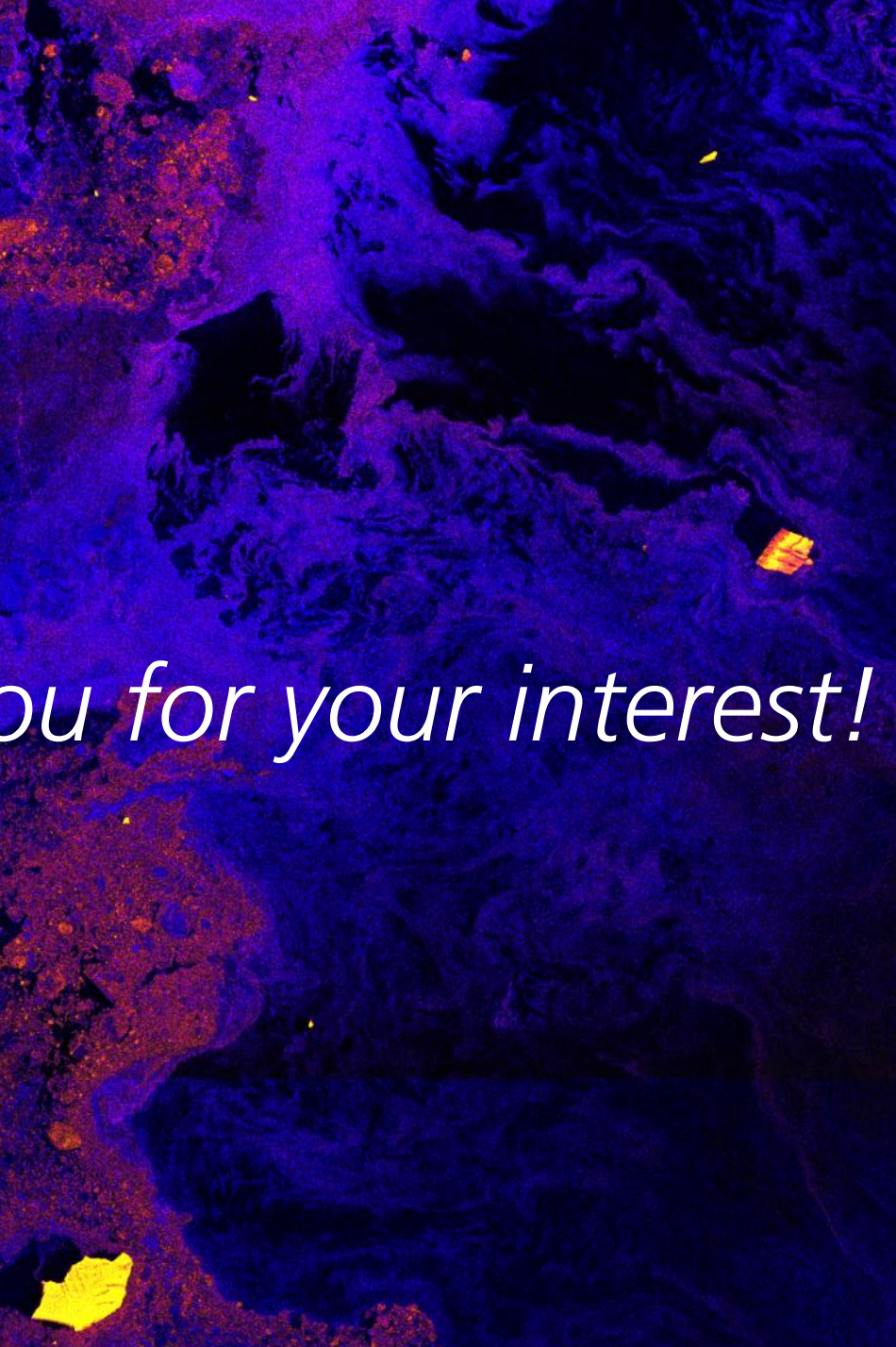

C.

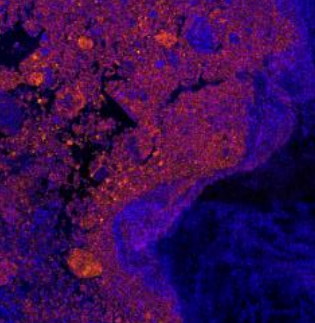

,
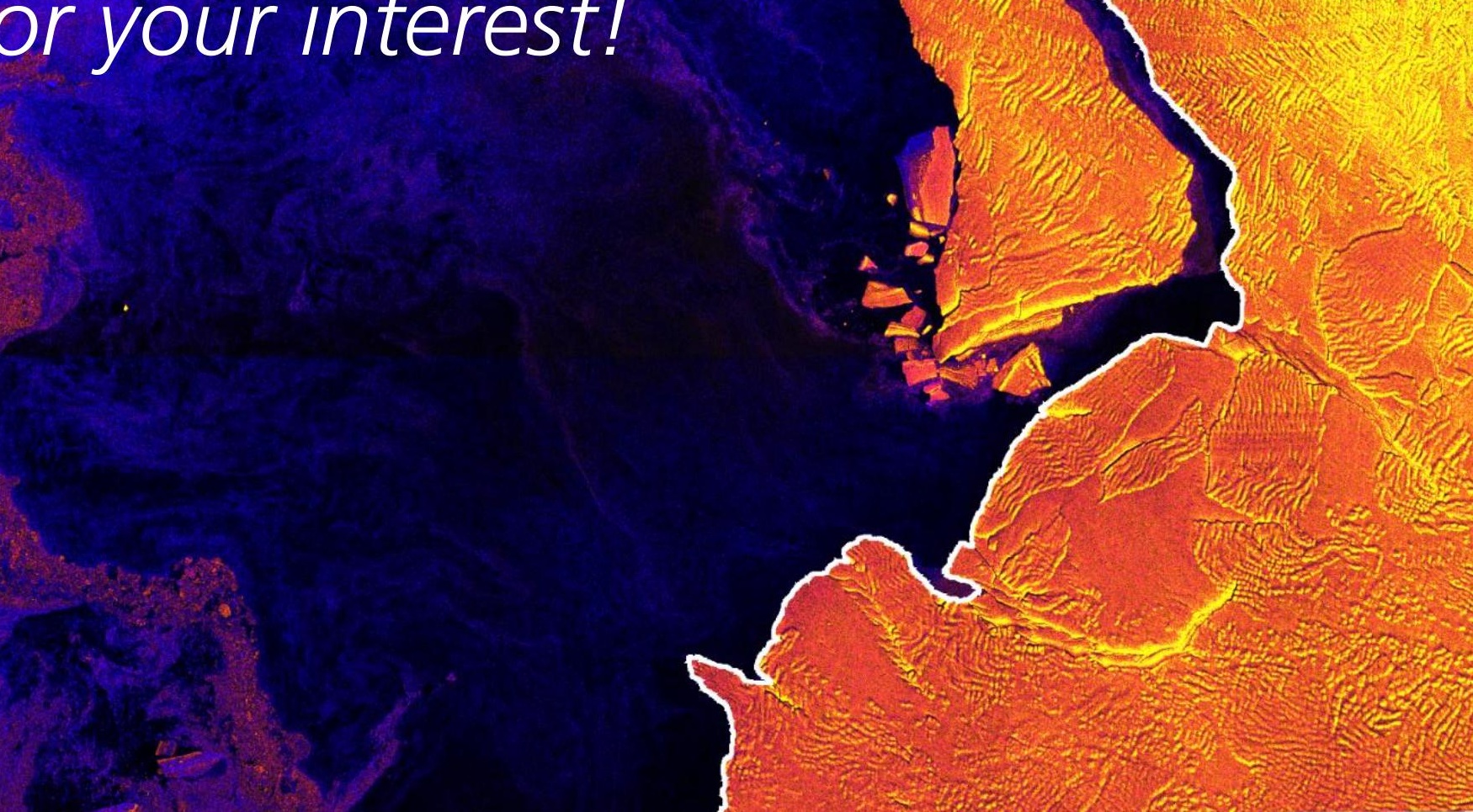


\section{References}

Baumhoer, C. A., Dietz, A. J., Kneisel, C., Paeth, H., \& Kuenzer, C. (accepted). Environmental Drivers of CircumAntarctic Glacier and Ice Shelf Front Retreat over the Last Two Decades. The Cryosphere. https://doi.org/10.5194/tc-2020-224

Baumhoer, C. A., Dietz, A. J., Kneisel, C., \& Kuenzer, C. (2019). Automated Extraction of Antarctic Glacier and Ice Shelf Fronts from Sentinel-1 Imagery Using Deep Learning. Remote Sensing, 11(21), 2529. https://doi.org/10.3390/rs11212529

Baumhoer, C., Dietz, A., Dech, S., \& Kuenzer, C. (2018). Remote Sensing of Antarctic Glacier and Ice-Shelf Front Dynamics-A Review. Remote Sensing, 10(9), 1445. https://doi.org/10.3390/rs10091445

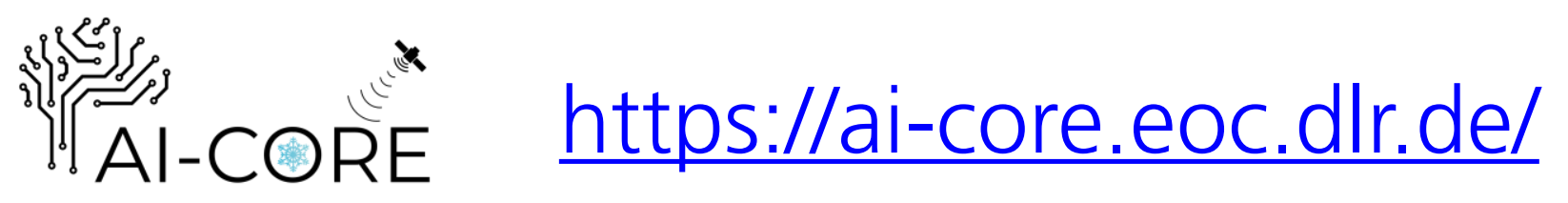

